\title{
Role of nuclear PTEN revealed
}

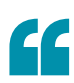

nuclear PTEN

interacts with

$\mathrm{APC} / \mathrm{C}$ and

enhances

its ability

to degrade

substrates.
In the cytoplasm, the tumour suppressor PTEN dephosphorylates phosphoinositide-3,4,5-trisphosphate to inhibit PI3K-AKT signalling, cell growth and cell survival. Nuclear PTEN also has a tumour-suppressive role, but its functions were unknown. Song et al. now show that nuclear PTEN interacts with the anaphasepromoting complex/cyclosome (APC/C), an E3 ubiquitin ligase that degrades components of the cell cycle machinery, to enhance its activity.

The authors immunoprecipitated PTEN from nuclear extracts and analysed samples by mass spectrometry. Four APC/C subunits - APC3, APC4, APC5 and APC7 - were associated with nuclear PTEN. PTEN silencing or overexpression in cells increased or decreased the level of APC/C substrates, respectively. Furthermore, recombinant PTEN increased the ability of APC/C to

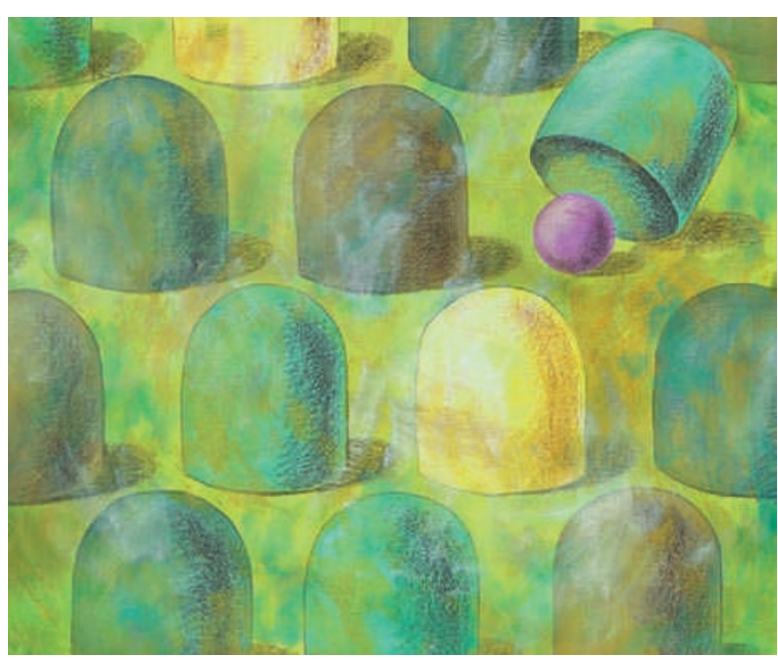

ubiquitylate cyclin B in vitro, and nuclear, but not cytoplasmic, PTEN immunoprecipitates could ubiquitylate cyclin B. Thus, nuclear PTEN interacts with APC/C and enhances its ability to degrade substrates.

APC/C activity is controlled by the binding of cell division cycle 20 (CDC20) in early mitosis and of CDH1 (also known as FZR1) in late mitosis and G1. As the ubiquitin ligase activity associated with PTEN is greater in interphase than in mitosis, the authors tested whether it influences APC/C-CDH1. Indeed, PTEN promotes the APC/C-CDH1 interaction and enhances the activity of this complex. Nuclear PTEN specifically regulates APC/C-CDH1, as a PTEN mutant that is excluded from the nucleus could not ubiquitylate cyclin $\mathrm{B}$, reduce the level of APC/C-CDH1 targets or interact with APC3. Thus, nuclear PTEN activates APC/C-CDH1 to regulate the degradation of its targets. Importantly, as CDH1-null cells were refractory to PTEN-mediated growth suppression, APC/C-CDH1 might regulate this biological property of PTEN.

The authors also asked whether the nuclear role of PTEN depends on its phosphatase activity. Overexpression of wild-type or phosphatase-inactive PTEN in PTEN-null cells reduced the levels of APC/C-CDH1 targets, such as polo-like kinase 1 (PLK1) and aurora $\mathrm{A}$, and enhanced the ubiquitin ligase activity of APC/ $\mathrm{C}-\mathrm{CDH} 1$. Thus, PTEN regulates
APC/C-CDH1 independently of its phosphatase activity.

So, does APC/C-CDH1 mediate the role of nuclear PTEN in tumour progression? Although a reduced level of PTEN or CDH1 decreases the tumour-suppressive activity of these proteins, a complete loss of these proteins leads to cellular senescence; this suppresses tumour progression even on PTEN loss. Importantly, overexpression of CDH1 in PTEN-null cells decreased the level of the senescence mediator p16. Loss of PTEN led to an increase in p16, the level of which was further increased on loss of both PTEN and CDH1. Thus, PTEN loss-induced senescence seems to be partly mediated by an APC/CCDH1-p16 pathway, whereas partial loss of this pathway and PTEN would favour tumorigenesis.

In short, nuclear PTEN positively regulates $\mathrm{APC} / \mathrm{C}-\mathrm{CDH} 1$, in a phosphatase-independent manner, to promote the downregulation of its targets and tumour suppression. This has implications for cancer therapy, as the authors show that loss of PTEN, but not inactivation of its phosphatase activity, sensitizes cells to pharmacological inhibition of PLK1 and aurora A, two APC/C-CDH1 substrates that are being investigated therapeutically.

Katharine H. Wrighton, Senior Editor,

Nature Reviews Molecular Cell Biology

ORIGINAL RESEARCH PAPER Song, M. S. et al Nuclear PTEN regulates the APC-CDH1 tumorsuppressive complex in a phosphatase-

independent manner. Cell 144, 187-199 (2011) 